\title{
Baricitinib-Studiendaten und Praxisalltag überzeugen mit guter Verträglichkeit
}

Bei der Behandlung der rheumatoiden Arthritis (RA) ist die Verträglichkeit eines der Hauptauswahlkriterien für die Therapie. Langzeitdaten und Erfahrung aus dem klinischen Alltag bestärken Arzt und Patient in der Therapieentscheidung.

\begin{abstract}
_ Für den Januskinase(JAK)-Inhibitor Baricitinib demonstrierte zu Beginn des Jahres eine Vollpublikation die Langzeitverträglichkeit über fünf Jahre [1]. Eine neue Auswertung [2] belegt die Verträglichkeit von Baricitinib über bis zu sieben Jahre.
\end{abstract}

\section{Langanhaltend gut verträglich}

Die im Rahmen des EULAR-Kongresses vorgestellten Daten einer integrierten Sicherheitsanalyse belegen die gute Verträglichkeit von Baricitinib über einen Zeitraum von bis zu sieben Jahren [2]. Die Datenauswertung von mehr als 3700 Patienten und über 10.100 Patientenjahren ergab keine neuen Sicherheitssignale.

Baricitinib zeigte auch ein gutes kardiovaskuläres Verträglichkeitsprofil. Die Inzidenzrate (IR) je 100 Patientenjahre für schwere kardiale Komplikationen (MACE) und tiefe Venenthrombosen oder Lungenembolien (DVT/ PE) lag jeweils bei 0,5/100 Patientenjahren'. Zu den häufigsten Infektionen zählte Herpes zoster mit einer Inzidenz von 3,3. In einer vorgestellten dezidierten Analyse zu Herpes zoster wurde die Inzidenz im Langzeitverlauf und in Abhängigkeit von Risikofaktoren ausgewertet. Dabei zeigte sich, dass die Inzidenz im Zeitverlauf über drei Jahre nicht zunahm. Die aufgetretenen Infektionen waren meist unkompliziert und betrafen nur ein Dermatom. Zu den ermittelten Risikofaktoren zählten die geographische

Gepoolte Daten aus neun randomisierten Studien mit Patienten, die mit Baricitinib (beide Dosierungen: $2 \mathrm{mg}$ und $4 \mathrm{mg}$ ) behandelt wurden.
Lage, mit einer höheren Inzidenz in Asien, und das Patientenalter (Hazard Ratio: 1,3). Das Risiko für Herpes zoster steigt im Allgemeinen mit dem Lebensalter an.

Aktuelle RWE-Daten gaben Aufschluss über die flexiblen Verschreibungsgewohnheiten von Baricitinib im klinischen Alltag: Je nach Analyse und Standort war der Einsatz des JAKInhibitors im Therapiealgorithmus, also nach csDMARD- oder bDMARD-Versagen, sehr unterschiedlich. Der Anteil der Patienten, die Baricitinib direkt nach csDMARD-Versagen und damit vor Biologika erhielten, variierte zwischen $18 \%$ und $60 \%$.

Das spiegelt wider, dass Baricitinib entsprechend der DGRh-Leitlinie flexibel im Therapiealgorithmus in der zweiten (nach csDMARD-Versagen) oder dritten (nach bDMARD-Versagen) Therapiephase eingesetzt werden kann. Neben der DGRh-Empfehlung erlaubt die Zulassung von Baricitinib in Monotherapie und in Kombination mit MTX einen flexiblen Einsatz, der an die Bedürfnisse des Patienten angepasst werden kann. Ärzte und Patienten haben so die Möglichkeit, eine Therapie mit oder ohne MTX zu wählen. Aktuellen Auswertungen zufolge wird diese Flexibilität im Praxisalltag genutzt: Etwa die Hälfte der Patienten erhielt demnach Baricitinib als Monotherapie ohne die Kombination mit MTX.

Gute Adhärenz und Verträglichkeit Neben der flexiblen Therapieinitiierung überzeugt Baricitinib auch im Therapieverlauf. Eine Auswertung des spanischen BIOBADASAR III-Registers fokussierte sich auf das Drug Survival unter den JAK-Inhibitoren Baricitinib und Tofacitinib, die in etwa gleich häufig verordnet und zusammen ausgewertet wurden. Nach 12-monatiger Behandlung hielten im Schnitt 82,5\% der Patienten an der Therapie mit einem der JAK-Inhibitoren fest. In der Gruppe der bDMARD-naiven Patienten führten alle Patienten die Therapie fort, sodass die Drug-Survival-Rate für diese Patientengruppe $100 \%$ betrug.

Die in Madrid präsentierten Versorgungsdaten zeigen ein mit den Studiendaten vergleichbares und damit konsistent gutes Sicherheitsprofil. Die meisten unerwünschten Arzneimittelwirkungen waren im Allgemeinen mild oder moderat, neue Sicherheitssignale wurden nicht berichtet.

\footnotetext{
Literatur

1. Smolen JS et al (2019) Safety Profile of Baricitinib in Patients with Active Rheumatoid Arthritis with over 2 Years Median Time in Treatment. J Rheumatol 46(1):7-18

2. Genovese MC et al (2019) Safety profile of Baricitinib for the treatment of rheumatoid arthritis up to 7 years: an updated integrated safety analysis. Ann Rheum Dis 78(suppl 2):308-309
}

rheuma plus $2019 \cdot 18: 120$ https://doi.org/10.1007/s12688019-0266-7 (c) Springer-Verlag GmbH Austria, ein Teil von Springer Nature 2019 